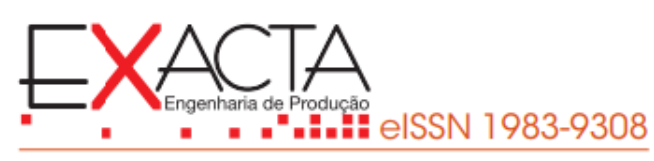

\title{
PRÁTICAS dE GESTÃo de PESSOAS NA CULTURA DE SEGURANÇA
}

\author{
PEOPLE MANAGEMENT PRACTICES IN THE SAFETY CULTURE
}

Recebido em: 17 nov. 2020

Aprovado em: 26 jan. 2021

Versão do autor aceita publicada online: 26 jan. 2021

Publicado online: 25 jun. 2021

\section{Como citar esse artigo - American Psychological Association (APA):}

Trentin, L. (2022, out./dez.). Práticas de gestão de pessoas na cultura de segurança. Exacta. 20(4), 969-995. https://doi.org/10.5585/exactaep.2022.18716.

Submeta seu artigo para este periódico $\beta$

Dados Crossmark 


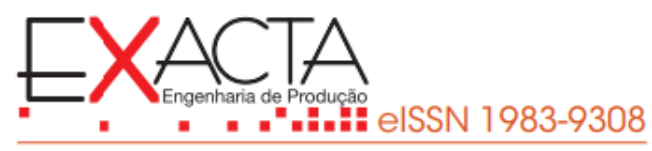

\title{
PRÁTICAS DE GESTÃO DE PESSOAS NA CULTURA DE SEGURANÇA
}

\author{
PEOPLE MANAGEMENT PRACTICES IN THE SAFETY CULTURE
}

${ }^{1}$ Doutor em Ciências Contábeis e Administração Universidade Regional de Blumenau - FURB. Itajaí, SC - Brasil. trentinluciano@yahoo.com.br

Recebido em: 17 nov. 2020

Aprovado em: 26 jan. 2021
Resumo: A gestão de pessoas assumiu um papel estratégico frequentemente utilizado no desenvolvimento de valores organizacionais compartilhados que afetam e influenciam atitudes e comportamentos das pessoas. Neste contexto, o presente artigo tem como objetivo analisar a influência das práticas de gestão de pessoas na cultura de segurança. A coleta de dados foi realizada por meio de uma survey descritiva, aplicada por intermédio da plataforma virtual Google Forms. A amostra foi composta por 204 respondentes de uma empresa do segmento de papel e celulose da região sul do Brasil, sendo coletada por acessibilidade e conveniência. Utilizou-se a análise de regressão múltipla para o tratamento e análise dos dados. Como resultados, evidenciou-se que as hipóteses $\mathrm{H} 2, \mathrm{H} 3, \mathrm{H} 4$ e $\mathrm{H} 5$ confirmaram que as práticas de gestão de pessoas: retenção, desenvolvimento, contrato e ambiente de trabalho influenciam o estabelecimento de uma cultura de segurança na organização. Em contrapartida, a hipótese $\mathrm{H} 1$ foi rejeitada, devido a prática de gestão de pessoas captação não ter demonstrado no teste confirmatório interferência na cultura de segurança.

Palavras-chave: Práticas de gestão de pessoas. Cultura de segurança. Gestão de pessoas.

Abstract: People management has taken on a strategic role often used in the development of shared organizational values that affect and influence people's attitudes and behaviors. In this context, this article aims to analyze the influence of people management practices on the safety culture. Data collection was performed through a descriptive survey, applied through the Google Forms virtual platform. The sample consisted of 204 respondents from a pulper and paper company in southern Brazil, being collected for accessibility and convenience. Multiple regression analysis was used for data treatment and analysis. As a result, it became evident that hypotheses $\mathrm{H} 2, \mathrm{H} 3, \mathrm{H} 4$ and $\mathrm{H} 5$ confirmed that people management practices: retention, development, contract and work environment influence the establishment of a safety culture in the organization. On the other hand, hypothesis $\mathrm{H} 1$ was rejected, due to the fact that the management of people enrollment did not demonstrate interference in the safety culture in the confirmatory test.

Keywords: Practices of people management. Safety culture. People management. 
1 introdução

Nas últimas décadas as organizações vêm vivenciando grandes mudanças em função dos avanços tecnológicos. Neste contexto torna-se importante ressaltar que as transformações introduzidas nos últimos anos sobre os processos de trabalho, modificou significativamente a relação entre as pessoas, os meios de produção e o ambiente de trabalho, e isso levou muitas organizações, conscientemente ou não, a maximizar o uso dos recursos da organização para proporcionar um ambiente de trabalho, saudável, seguro e produtivo (Araújo, Santos, \& Mafra, 2006).

Contudo as mudanças não se limitam às estruturas físicas e produtos, mas especialmente sobre os padrões comportamentais ou culturais. Assim, para que as organizações se adaptem ao novo cenário, torna-se necessário pensar em seus colaboradores, não como simples "recursos humanos", mas sim, como o "capital humano" da organização (Spinelli, 2015).

A valorização do capital humano nas organizações com a criação, transferência e aplicação do conhecimento, assume um papel fundamental na era da gestão do conhecimento e crescente competitividade entre as organizações. Deste modo, estrategicamente, torna-se essencial que a área de Gestão de Pessoas [GP], através de suas políticas e práticas, assuma a responsabilidade pela criação de competências organizacionais que contribuam para melhor gerir atitudes e comportamentos das pessoas (Bohlander \& Snell, 2009; Christopher, 2019; Demo, Fogaça, \& Costa, 2017).

Macarenco (2006) compreende que atrair, desenvolver e reter talentos possibilita a área de gestão pessoas o reconhecimento de parceira estratégica pelas organizações. Youndt e Snell (2004) mencionam que as práticas de gestão de pessoas, captação e desenvolvimento, podem contribuir para aperfeiçoar o capital humano das organizações, melhorando assim o ambiente de trabalho.

Ambientes de trabalho adequados, requerem maior responsabilidade das organizações, desde o emprego inteligente de políticas, práticas e normativas de gestão de pessoas destinadas a estimular os colaboradores para que sintam motivados a produzir, até a satisfação de seus desejos e necessidades em ambientes favoráveis (Khoo, Hussin, \& Abdullah, 2018). Assim, Coutinho, Maximiano e Limongi-França (2010) expõem a necessidade da área de gestão de pessoas desenvolver ações e programas relacionados à Cultura de Segurança [CS], visto que estes destinam-se a harmonizar a relação entre o colaborador e seu ambiente laboral (Dejours, 2012).

A cultura de segurança é definida por Reason (2016) como um conjunto de princípios éticos que regem as interações organizacionais, norteando aspectos morais de conduta, para produzir normas comportamentais. Segundo Nilsson e Vänje (2018) a cultura de segurança é uma 
área de conhecimento complexa, interdisciplinar e orientada para a prática, devendo ser difundida entre os diferentes grupos de interesses e imperiosamente inserida nos planos estratégicos das organizações (Araújo, 2005; Pinho, 2006).

Nesse sentido, Dutra (2008) menciona que as políticas e práticas de gestão de pessoas contribuem para a concitação dos objetivos estratégicos das organizações e as expectativas das pessoas. Jabbour e Santos (2009) evidenciam que as políticas e práticas de gestão de pessoas podem influenciar o comportamento e as atitudes das pessoas.

Gerenciar o comportamento e as atitudes das pessoas, é parte integrante do papel exercido pelos gestores de pessoas, tornando-se um arcabouço para a criação de valores e desenvolvimento de ambientes laborais benéficos, favorecendo o desenvolvimento de uma cultura de segurança duradoura (Oliveira, 2014). Corrigan et al. (2018) mencionam que as organizações devem identificar as principais políticas, procedimentos e práticas de gestão de pessoas necessárias para se condicionar uma cultura de segurança mais longeva.

De acordo com Araújo (2005), faz-se necessário maior esforço da organização em desenvolver planos estratégicos de gestão de pessoas, convertê-los em planos de ação e implementá-los, assim integrando as práticas de gestão de pessoas na criação ou desenvolvimento de uma cultura de segurança em todos os níveis hierárquicos e métodos de trabalho.

Para que as práticas de gestão de trabalho permeiem todos os níveis hierárquicos e os métodos de trabalho, destaca-se Delery e Roumpi (2017), os quais evidenciam que as práticas de gestão de pessoas devem estar alinhadas com as diretrizes da organização, para que os colaboradores não construam seu próprio entendimento a respeito destas, fazendo-se necessário constituir uma área de gestão de pessoas com princípios técnicos, relacionais e estratégicos consistentes.

Diante do exposto, o presente artigo tem como objetivo analisar a influência das práticas de gestão de pessoas na cultura de segurança. Segundo Lima, Souza e Lima (2018) e Choudhry, Fang e Mohamed (2007) a implementação de políticas e práticas de gestão e pessoas é de fundamental importância para que se consiga o envolvimento das pessoas na compreensão e aceitação da cultura de segurança. Migueles, Lafraia e Costa (2007) expõem a necessidade de desenvolver políticas e práticas e gestão de pessoas na institucionalização da cultura de segurança.

Esta pesquisa enquadra-se, quanto aos objetivos, na categoria de pesquisa descritiva, no que tange aos procedimentos, prevê a abordagem de levantamento survey, utilizando como ferramenta principal de análise a abordagem quantitativa, estando organizado da seguinte forma: a segunda seção é uma revisão teórica das práticas de gestão de pessoas e da cultura de 
segurança, a terceira seção expressa a metodologia utilizada, a quarta seção apresenta e discute os resultados obtidos e por fim são feitas as considerações finais e as recomendações para trabalhos futuros.

\section{Práticas de gestão de pessoas}

Compreende-se que para crescer e sustentar as posições conquistadas pelas organizações, é preciso antecipar-se às mudanças frequentes no ambiente de negócios e adotar respostas ágeis às decisões relacionadas à gestão de pessoas (Fitz-Enz, 2010). Essa conjuntura demonstra a transição do papel exercido pela área de gestão de pessoas, distanciando-se das questões mais rotineiras e de relações trabalhistas, para uma orientação estratégica, essencialmente gerenciada por políticas e práticas efetivas de gestão de pessoas (Kianto, Sáenz, \& Aramburu, 2017; Pavlov et al., 2017; Trentin, Duarte, \& Santos, 2018).

Nesse contexto, destaca-se que as práticas de gestão de pessoas (Tabela 1) apresentam vários conceitos, os quais refletem as várias definições sobre a temática. De acordo com Robbins (2004) as práticas e processos de gestão de pessoas destinam-se a prover as organizações com profissionais capacitados e perpetuar o alto desempenho dos colaboradores por intermédio de um "planejamento estratégico de recursos humanos, recrutamento ou redução do quadro, seleção, orientação, treinamento, avaliação do desempenho, remuneração, e benefícios, segurança e saúde, e ao lidar com as questões contemporâneas de administração de recursos humanos" (p.140).

\section{Tabela 1}

Práticas de Gestão de Pessoas

\begin{tabular}{|c|c|c|}
\hline $\begin{array}{l}\text { Tipo de } \\
\text { prática }\end{array}$ & Conceito da prática & $\begin{array}{l}\text { Conjunto de ações } \\
\text { Adaptado de Fiuza (2008); Limongi- } \\
\text { França (2008); Silva (2013). }\end{array}$ \\
\hline Captação & $\begin{array}{l}\text { Criar estratégias de recrutamento e seleção de } \\
\text { profissionais dispostos a se envolver e se } \\
\text { comprometer com as atividades desenvolvidas na } \\
\text { organização (Freitas, 2014). }\end{array}$ & $\begin{array}{l}\text { Recrutamento interno e externo; Seleção } \\
\text { de pessoal; Programa de integração; } \\
\text { Entrevista de desligamento. }\end{array}$ \\
\hline $\begin{array}{l}\text { Desenvolvi } \\
\text {-mento }\end{array}$ & $\begin{array}{l}\text { Promover e ampliar a aquisição de competências, } \\
\text { regras, conceitos ou atitudes que resultem em } \\
\text { melhor sinergia entre as características das pessoas e } \\
\text { as demandas dos papéis organizacionais exercidos } \\
\text { (Freitas, 2014; Milkovich, 2000). }\end{array}$ & $\begin{array}{l}\text { Treinamento; On the job training; } \\
\text { Educação formal; Identificação de } \\
\text { competências; Plano de } \\
\text { desenvolvimento individual [PDI]; Plano } \\
\text { de desenvolvimento coletivo [PDC]. }\end{array}$ \\
\hline Retenção & $\begin{array}{l}\text { Programas de recompensa financeira ou não, com o } \\
\text { objetivo de retribuir, valorizar e estimular } \\
\text { comportamentos e ações de pessoas que agregam } \\
\text { valor à organização (Hipólito, 2000). }\end{array}$ & $\begin{array}{l}\text { Remuneração fixa e variável; Programa } \\
\text { de participação nos resultados [PPR]; } \\
\text { Avaliação de desempenho; Plano de } \\
\text { benefícios. }\end{array}$ \\
\hline
\end{tabular}




\begin{tabular}{c|l|l}
\hline $\begin{array}{c}\text { Tipo de } \\
\text { prática }\end{array}$ & \multicolumn{1}{|c}{ Conceito da prática } & \multicolumn{1}{c}{$\begin{array}{c}\text { Conjunto de ações } \\
\text { Adaptado de Fiuza (2008); Limongi- } \\
\text { França (2008); Silva (2013). }\end{array}$} \\
\hline $\begin{array}{c}\text { Contrato } \\
\text { de } \\
\text { trabalho }\end{array}$ & $\begin{array}{l}\text { Gerenciar os processos de controle administrativo e } \\
\text { jurídico, no que diz respeito às relações de trabalho } \\
\text { entre empregados e empregadores (Júnior, Morais, \& } \\
\text { Teixeira, 2013). }\end{array}$ & $\begin{array}{l}\text { Gerenciamento de procedimentos } \\
\text { administrativos e legais; Pagadoria; } \\
\text { Negociações sindicais; Relações } \\
\text { trabalhistas. }\end{array}$ \\
\hline $\begin{array}{c}\text { Ambiente } \\
\text { de }\end{array}$ & $\begin{array}{l}\text { Ambientes constituídos no comportamento das } \\
\text { pessoas e no engajamento com as práticas } \\
\text { organizacionais estabelecidas, propícias ao } \\
\text { desenvolvimento humano, bom relacionamento } \\
\text { interpessoal e bem-estar dentro e fora do ambiente } \\
\text { de trabalho (McClean \& Collins, 2011; Trentin et al., } \\
\text { 2016). }\end{array}$ & $\begin{array}{l}\text { Estímulo na tomada de decisão; } \\
\text { Comualidade de vida no trabalho [QVT]; } \\
\text { Gestão de saúde e segurança } \\
\text { ocupacional [GSSO]. }\end{array}$ \\
\hline
\end{tabular}

Fonte: Elaborado pelo autor (2020).

Dutra (2008) menciona que a gestão de pessoas impõe pensar a atuação da área como "um conjunto de políticas e práticas que possibilitem a conciliação de expectativas entre a organização e as pessoas para que ambas possam realizá-las ao longo do tempo" (p.17). Para o autor, as políticas são as diretrizes, os princípios que guiam o comportamento das pessoas nas organizações.

A gestão de pessoas para Jabbour, Santos e Nagano (2009) é um composta de práticas concebidas para direcionar o modo de se comportar das pessoas concomitantemente com os propósitos organizacionais estabelecidos, com reciprocidade de influência entre a área de gestão de pessoas e as demais áreas da organização.

Schaurich (2014) evidencia que as políticas de gestão de pessoas são desdobradas em uma série de práticas diretamente relacionadas a elas. A autora destaca que as práticas de gestão de pessoas correspondem aos diversos procedimentos, métodos e técnicas utilizadas para a implementação das decisões organizacionais. Para Collins e Smith (2006) as ações de gestão de pessoas devem incluir uma combinação a longo prazo de práticas de captação, retenção, desempenho e desenvolvimento, regidos de forma transparente por acordos recíprocos

Mediante os conceitos apresentados, entende-se que a gestão de pessoas deve se moldar às necessidades da organização. Essas necessidades estão em constantes transformações, devendo as práticas de gestão de pessoas ajustar-se com o desenvolvimento das organizações. Sobre este ajuste, Jabbour et al. (2012) expressam que em uma perspectiva contemporânea e estratégica, a gestão de pessoas é conhecida como projeto e um arranjo de práticas de gestão orientadas para o aperfeiçoamento da eficácia organizacional na demanda por convergências entre as políticas de gestão de pessoas e um ambiente organizacional propício.

Independentemente da gestão de pessoas resultar da implementação de práticas diferenciadas em inúmeras localidades, em consequências não só da cultura, mas também das 
condições socioeconômicas e políticas, o efetivo exercício do papel desempenhado pela área de gestão de pessoas inevitavelmente resulta em um ambiente laboral positivo em todas as organizações (Kianto et al., 2017). Freitas (2014) e Freitas, Jabbour e Santos (2011) destacam as adversidades para harmonizar as demandas da organização à realidade do ambiente vivenciado, como uma das principais demandas a ser efetivamente gerida pelos gestores responsáveis pela área de gestão de pessoas.

A concordância entre as práticas de gestão de pessoas e as estratégias organizacionais, para dirimir qualquer possibilidade de desalinhamento, requer que os gestores considerem a conjuntura na qual então inseridos, superando assim possíveis disfunções relacionais entre as pessoas (Freitas, 2014; Freitas, Jabbour, \& Santos, 2011).

A respeito das relações existente nas organizações, Jabbour et al. (2012) e Visser (2010) compreendem que as atitudes e comportamento das pessoas, são influenciados pela configuração do conjunto de práticas de gestão de pessoas adotadas nas organizações e, por intermédio destas, também o ambiente de trabalho (Jabbour et al., 2012; Visser, 2010).

\subsection{Cultura de Segurança}

Concebido a partir do primeiro relatório técnico elaborado pelo International Nuclear Safety Advisory Group [INSAG] no ano de 1988, o conceito de "cultura de segurança" foi elaborado mediante uma série de fatores organizacionais, através do resultado da análise das origens do acidente da usina nuclear de Chernobyl, na Ucrânia, em 1986 (Agência Internacional de Energia Atômica [AIEA], 1991). O conceito de cultura de segurança foi elaborado como: "o conjunto de características e atitudes das organizações e dos indivíduos, que garante que a segurança de uma planta nuclear, pela sua importância, terá a maior prioridade" (INSAG, 1988, p.84).

Conforme Helmer (2002), Pidgeon (1991) e Turner et al. (1989) a cultura de segurança expressa uma singularidade quanto ao conjunto de preceitos e regras institucionais e aos fundamentos constituintes da consciência das pessoas dentro de uma organização, com propósito de restringir a exposição dos stakeholders as condições classificadas como não salubres. Nessa conjuntura, onde existe cultura de segurança constituída, a maneira de se comportar dos colaboradores (agir e reagir) em relação à segurança se manifesta e perdura.

De acordo com Cooper (2000) a cultura de segurança é determinada como consequência das interações dinâmicas entre três aspectos: 1) os pressupostos básicos e valores; 2) práticas coletivas; e 3) estrutura da organização. Conforme o autor, as interações dinâmicas entre esses aspectos podem variar em intensidade e no tempo. Por exemplo, modificações nas estruturas 
organizacionais podem demorar a influenciar os valores dos colaboradores da organização. A gestão da cultura de segurança pode interagir com as outras perspectivas presentes no conjunto de hábitos e valores organizacionais, por intermédio das práxis organizacionais, constituindo assim a cultura de segurança (Cooper, 2000).

Ainda de acordo com Cooper (2000), com estes aspectos é possível mensurar a cultura de segurança de forma relevante em diferentes estágios organizacionais. A Figura 1 demonstra as interações entre os três aspectos presentes na cultura de segurança.

\section{Figura 1}

Modelo de Interações Recíprocas de Cultura de Segurança.

\begin{tabular}{|c|}
\hline Atitudes e \\
percepções \\
(indivíduo) \\
\hline
\end{tabular}

Fatores subjetivos

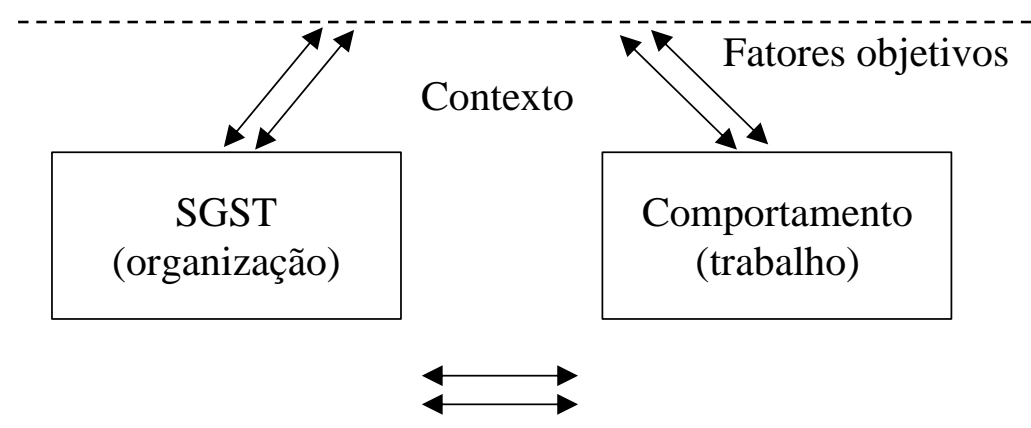

Fonte: Adaptado de Cooper (2000, p.119).

As atitudes e percepções são como as pessoas reconhecem a organização, sendo relacionadas ao indivíduo, encontrando-se no campo subjetivo e considerados fatores não observáveis; os comportamentos e as ações são o que as pessoas fazem na organização e estão relacionadas ao trabalho; e o sistema de Gestão de Saúde e Segurança Ocupacional [GSSO] é formado pelas políticas, procedimentos, sistemas de controle, fluxo de informações e está relacionado à organização. Os aspectos comportamento e ação e o sistema de GSSO são considerados objetivos e passíveis de serem observados (Cooper, 2000).

Assim, a cultura de segurança foi caracterizada como o mais importante preditor do comportamento organizacional em saúde e segurança ocupacional, incluindo a demonstração pela administração de comprometimento com a saúde e segurança, educação e conhecimento 
da força de trabalho, eficácia do processo de gestão e envolvimento e comprometimento dos colaboradores (Carder \& Regan, 2003).

Rodrigues (2011) menciona que os aspectos-chave (Tabela 2) da cultura de segurança devem ser compreendidos como a percepção partilhada de normas, valores, procedimentos e práticas de segurança.

Tabela 2

Aspectos-Chave da Cultura de Segurança

\begin{tabular}{l|l}
\hline Empenho da gestão & $\begin{array}{l}\text { Gerar níveis mais elevados de motivação e preocupação relativamente à segurança } \\
\text { e saúde no trabalho em toda a organização. É indicado pela proporção de recursos } \\
\text { (tempo, dinheiro, colaboradores) e apoio atribuídos à gestão da saúde e segurança } \\
\text { e pelo estatuto concedido à saúde e segurança em oposição, por exemplo, à } \\
\text { produção e custos. }\end{array}$ \\
\hline $\begin{array}{l}\text { Os gestores têm de ser vistos e darem o exemplo relativamente à saúde e segurança. } \\
\text { Os bons gestores aparecem regularmente no 'local de trabalho', falam sobre a saúde } \\
\text { e segurança e demonstram visivelmente o seu empenho através das suas ações (por } \\
\text { exemplo, parar as atividades para resolver problemas). }\end{array}$ \\
\hline $\begin{array}{l}\text { Boa comunicação entre } \\
\text { todos os níveis de } \\
\text { colaboradores }\end{array}$ & $\begin{array}{l}\text { Numa cultura positiva, as questões sobre saúde e segurança devem fazer parte dos } \\
\text { debates de trabalho diários. A gestão deve ouvir ativamente as sugestões dos } \\
\text { colaboradores e desenvolver comportamentos seguros. }\end{array}$ \\
\hline $\begin{array}{l}\text { Participação ativa dos } \\
\text { colaboradores }\end{array}$ & $\begin{array}{l}\text { A participação na segurança é importante para criar responsabilidade pela segurança } \\
\text { a todos os níveis e explorar os conhecimentos úteis que os colaboradores possuem } \\
\text { do seu próprio trabalho. }\end{array}$ \\
\hline
\end{tabular}

Fonte: Adaptado de Rodrigues (2011, p.44).

Reason (2006) estabelece que a cultura de segurança de uma organização é definida pelo comportamento do indivíduo e das equipes, além do estilo e a perfeição da gestão de saúde e segurança ocupacional nas organizações. Nessa perspectiva, Blair e O'Toole (2010) retratam que a cultura de segurança é "um fator primordial e um preditor da melhoria do desempenho de segurança" (p.30) e Gonçalves (2011) complementa menciona que o conceito de cultura de segurança é definido pelas organizações que asseguram o bem-estar no desempenho das ocupações laborais em ambientes saudáveis. Nesse sentido, Pranee (2010) menciona que a qualidade de vida no trabalho abrange a cultura de segurança, por intermédio do bem-estar, desenvolvimento profissional e aperfeiçoamento do ambiente e trabalho.

Reiman e Rollenhagen (2014) confirmaram que a segurança deve conciliar aspectos humanos, comportamentais, estruturais e aspectos externos, como os aspectos sociais e ambiente econômico e não somente uma mera tecnologia (Sgourou et al., 2014). Assim, a cultura de segurança, pode ser estabelecida como acontecimentos ligados as áreas de segurança e os 
ambientes laborais, realizados em um local definido, compreendendo a interação entre os valores e as crenças e a estrutura organizacional, formalizada ou não (Alves, 2014).

O conceito de cultura de segurança adotado por Reason (2016), é definido como o conjunto de valores compartilhados e crenças que interagem com as estruturas organizacionais e com os sistemas de controle, para produzir normas comportamentais. Além disso, o autor defende quatros aspectos principais no processo de cultura de segurança: (i) cultura de reporte, na qual as pessoas percebem a importância e a confiança de se relatarem acidentes ocorridos em sua rotina; (ii) cultura de aprendizagem contínua; (iii) cultura de justiça, que gera confiança para que as pessoas relatem livremente ocorrências anormais que possam ocorrer; e, por fim, (iv) a flexibilidade, a qual se traduz na capacidade de a organização passar a considerar uma estrutura horizontalizada propiciando mais autonomia.

Diante das reflexões apontadas sobre as práticas de gestão de pessoas e a cultura de segurança nos ambientes laborais, a partir do referencial teórico, foram constituídas cinco hipóteses, retratadas na Tabela 3:

\section{Tabela 3}

\section{Hipóteses da pesquisa}

$H_{1}$ : A prática de captação influencia a cultura de segurança;

$\mathrm{H}_{2}$ : A prática de retenção influencia a cultura de segurança;

$H_{3}$ : A prática de desenvolvimento influencia a cultura de segurança;

$H_{4}$ : A prática de contrato de trabalho influencia a cultura de segurança;

$H_{5}$ : A prática de ambiente de trabalho influencia a cultura de segurança.

Fonte: elaborado pelo autor (2020).

As cinco hipóteses constituídas a partir do resumo bibliográfico foram experienciadas na prática e retratadas na próxima seção.

\section{Metodologia}

A pesquisa caracteriza-se por quantitativa por utilizar-se de indicadores como forma de analisar dimensões, por meio da captação de dados que indicam o funcionamento das instituições (Lindfelt et al., 2018).

O questionário foi composto de 65 perguntas e por um conjunto de componentes, com foco em identificar a influência das práticas de gestão pessoa na cultura de segurança, sendo a variável gestão de pessoas por intermédio dos componentes: captação, retenção, desenvolvimento, contrato de trabalho e ambiente de trabalho; e a variável cultura de segurança 
por intermédio dos componentes: empenho da gestão, comunicação, participação dos colaboradores, formação e informação, motivação, observação dos procedimentos de segurança, organização e aprendizagem, mudança organizativa de transição.

Cada pergunta do questionário foi formulada com base em Foroni (2014) e Freitas (2014), que envolveram em suas pesquisas o desenvolvimento de instrumentos para mensurar a gestão de pessoas e Rodrigues (2011) na cultura de segurança. Utilizou-se a escala Likert de cinco opções para cada assertiva do questionário, alternando entre (1) Nunca e (5) Sempre com as afirmativas que compuseram as questões.

Para a obtenção dos dados foi realizado uma survey com corte transversal e utilização de questionário estruturado disponibilizados para preenchimento via web, através do site especializado em coleta de dados google forms. A amostra foi composta por 204 respondentes ( $22,57 \%$ do número total de colaboradores) de uma empresa do segmento de papel e celulose da região sul do Brasil, formada por gestores, operadores, manutentores e técnicos, sendo coletada por acessibilidade e conveniência no período de março a junho de 2020.

O processo de análise começa com uma revisão e organização dos dados para inspecionar a integridade e coerência do banco de dados, por intermédio do software estatístico SPSS ${ }^{\circledR}$ (Statistical Package for the Social Sciences) versão 22. Procedeu-se com a estatística descritiva para melhor compreender as características da amostragem. Por intermédio da amostragem, é possível analisar e obter características específicas da população (Hair Jr. et al., 2009). Em seguida foi realizada a comparação entre os valores médios por área de trabalho, por meio do teste de Wilcoxon-Mann-Whitney (U). Segundo Campbell e Skillings (1985) o teste de Wilcoxon-MannWhitney $(U)$ é indicado para testar a diferença entre dois grupos não pareados em uma única variável ordinal. Utilizou-se também a regressão linear múltipla para verificar quais as melhores variáveis independentes (práticas de gestão de pessoas) para uma determinada variável dependente (cultura de segurança). De acordo com Fávero et al. (2009) a regressão multivariada quantifica a associação entre uma ou mais variáveis preditoras e uma variável de resultado. Finalizados os procedimentos metodológicos, apresenta-se as análises dos resultados encontrados na próxima seção.

\section{Resultados da pesquisa}

A fim de melhor compreender as características da amostra, investigou-se aspectos demográficos. A Tabela 4 apresenta uma predominância de respondentes do sexo masculino $(69,1 \%)$ entre os participantes da pesquisa. Em relação à distribuição dos respondentes por 
função, verifica-se que $61,8 \%$ ocupam cargos operacionais. A maioria dos participantes da pesquisa $(74,0 \%)$ possui ensino médio como formação educacional.

\section{Tabela 4}

Distribuição dos Respondentes

\begin{tabular}{c|c|c|c|c|c}
\multirow{2}{*}{ Gênero } & \multicolumn{2}{|c|}{ Masculino } & \multicolumn{2}{c}{ Feminino } \\
\cline { 2 - 6 } & 141 & 69,1 & & 63 & 30,9 \\
\hline Escolaridade & Frequência & Percentual & Função & Frequência & Percentual \\
\hline Especialização & 8 & 3,9 & Gestor & 8 & 3,9 \\
\hline Ensino superior & 22 & 10,8 & Administrativo & 23 & 11,3 \\
\hline Ensino médio & 151 & 74,0 & Manutentor & 39 & 19,1 \\
\hline Ensino fundamental & 23 & 11,3 & Operador & 126 & 61,8 \\
\hline Total & - & - & Técnico & 9 & 3,9 \\
\hline
\end{tabular}

Fonte: Dados da pesquisa (2020).

Destaca-se que o tempo médio de permanência dos respondentes na organização é de 6,15 anos, enquanto o tempo médio de permanência na atual função é de 4,39 anos. Dos participantes da pesquisa, $64,7 \%$ são casados e $35,3 \%$ solteiros. Em relação a distribuição dos respondentes por área de trabalho, 50,0\% estão lotados na área produtiva, 20,6\% na área de manutenção, 17,7\% na área logística, 10,3\% na área administrativa e 1,5\% na área florestal.

Após finalizada a análise da distribuição dos respondentes, foi realizada uma análise descritiva da amostra. É importante destacar que análise descritiva da amostra foi estratificada por área de trabalho com base nos resultados obtidos, a fim de identificar se as práticas de gestão de pessoas e cultura de segurança permeiam todas as áreas da organização de maneira equânime. A Tabela 5 mostra os valores médios de cada componentes por área de trabalho pesquisada. Como podemos observar, o maior valor médio total obtido entre as práticas de gestão de pessoas é o componente captação com valor médio de 4,0, enquanto a menor pontuação média de 3,5 pertence à dimensão ambiente de trabalho. Em relação a cultura de segurança, verifica-se que o valor médio total de todos os componentes ficou acima de 4 . 
Tabela 5

Análise Descritiva

\begin{tabular}{|c|c|c|c|c|c|c|c|c|c|c|c|c|c|}
\hline & & \multicolumn{12}{|c|}{ Área de Trabalho } \\
\hline & \multirow{2}{*}{ Componentes } & \multicolumn{2}{|c|}{$\begin{array}{c}\text { Administra- } \\
\text { tiva }\end{array}$} & \multicolumn{2}{|c|}{ Logística } & \multicolumn{2}{|c|}{ Manutenção } & \multicolumn{2}{|c|}{ Operacional } & \multicolumn{2}{|c|}{ Florestal } & \multicolumn{2}{|c|}{ Total } \\
\hline & & $\begin{array}{l}\text { Médi } \\
\text { a }\end{array}$ & DP & $\begin{array}{l}\text { Médi } \\
\text { a }\end{array}$ & DP & $\begin{array}{l}\text { Médi } \\
\text { a }\end{array}$ & DP & $\begin{array}{l}\text { Médi } \\
\text { a }\end{array}$ & DP & $\begin{array}{l}\text { Médi } \\
\text { a }\end{array}$ & DP & $\begin{array}{c}\text { Médi } \\
\text { a }\end{array}$ & DP \\
\hline \multirow{6}{*}{ 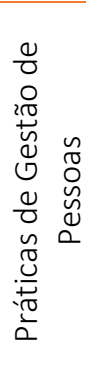 } & Captação & 4,2 & 0,486 & 3,9 & 0,442 & 3,8 & 0,524 & 4,0 & 0,396 & 4,1 & 0,115 & 4,0 & 0,453 \\
\hline & Retenção & 4,0 & 0,605 & 3,7 & 0,609 & 3,6 & 0,770 & 3,9 & 0,588 & 3,5 & 0,833 & 3,8 & 0,644 \\
\hline & Desenvolvimento & 4,1 & 0,427 & 3,9 & 0,468 & 3,9 & 0,487 & 4,0 & 0,467 & 3,6 & 0,529 & 3,9 & 0,471 \\
\hline & $\begin{array}{l}\text { Contrato de } \\
\text { trabalho }\end{array}$ & 4,1 & 0,457 & 3,8 & 0,390 & 3,8 & 0,464 & 3,8 & 0,426 & 3,8 & 0,346 & 3,9 & 0,433 \\
\hline & $\begin{array}{l}\text { Ambiente de } \\
\text { trabalho }\end{array}$ & 3,8 & 0,695 & 3,4 & 0,523 & 3,4 & 0,665 & 3,5 & 0,518 & 3,3 & 0,643 & 3,5 & 0,578 \\
\hline & Total & 4,0 & 0,408 & 3,7 & 0240 & 3,7 & 0,464 & 3,8 & 0,333 & 3,7 & 0378 & 3,8 & 0,383 \\
\hline \multirow{9}{*}{ 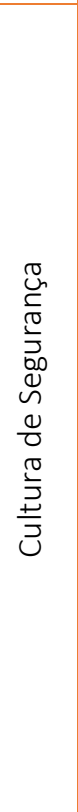 } & $\begin{array}{l}\text { Empenho da } \\
\text { gestão }\end{array}$ & 4,2 & 0,710 & 4,1 & 0,701 & 4,0 & 0,776 & 4,3 & 0,618 & 3,6 & 1,058 & 4,2 & 0,691 \\
\hline & comunicação & 4,0 & 0,753 & 3,9 & 0,807 & 3,9 & 0,731 & 4,0 & 0,757 & 3,7 & 1,155 & 4,0 & 0,761 \\
\hline & $\begin{array}{l}\text { Participação dos } \\
\text { colaboradores }\end{array}$ & 4,0 & 0,871 & 4,0 & 0,864 & 3,8 & 0,835 & 4,3 & 0,699 & 3,6 & 0,962 & 4,1 & 0,797 \\
\hline & $\begin{array}{l}\text { Formação / } \\
\text { Informação }\end{array}$ & 4,4 & 0,613 & 4,2 & 0,808 & 4,2 & 0,603 & 4,4 & 0,710 & 3,8 & 1,155 & 4,3 & 0,706 \\
\hline & Motivação & 4,3 & 0,720 & 4,2 & 0,800 & 4,3 & 0,580 & 4,4 & 0,619 & 3,8 & 1,155 & 4,3 & 0,670 \\
\hline & $\begin{array}{l}\text { Observação dos } \\
\text { procedimentos } \\
\text { de segurança }\end{array}$ & 4,3 & 0,809 & 4,3 & 0,729 & 4,2 & 0,671 & 4,3 & 0,663 & 3,6 & 1,072 & 4,3 & 0,698 \\
\hline & $\begin{array}{l}\text { Organização da } \\
\text { aprendizagem }\end{array}$ & 4,7 & 0,372 & 4,4 & 0,822 & 4,5 & 0,690 & 4,6 & 0,612 & 3,7 & 1,155 & 4,5 & 0,668 \\
\hline & $\begin{array}{l}\text { Mudança } \\
\text { organizativa e } \\
\text { gestão de } \\
\text { transição }\end{array}$ & 4,3 & 0,766 & 4,3 & 0,766 & 4,1 & 0,725 & 4,4 & 0,656 & 3,7 & 1,155 & 4,3 & 0,716 \\
\hline & Total & 4,2 & 0,609 & 4,1 & 0,677 & 4,1 & 0,578 & 4,3 & 0,565 & 3,6 & 1,095 & 4,2 & 0,607 \\
\hline \multicolumn{2}{|c|}{ № de Respondentes } & \multicolumn{2}{|c|}{$=1$} & \multicolumn{2}{|c|}{36} & \multicolumn{2}{|c|}{42} & \multicolumn{2}{|c|}{102} & & \multicolumn{2}{|c|}{04} \\
\hline
\end{tabular}

Fonte: Dados da pesquisa (2020).

Verificou-se também se há alternância entre as variáveis por meio dos valores médios atribuídos às práticas de gestão de pessoas e a cultura de segurança por área de trabalho pesquisada. Os dados foram testados para normalidade, por meio do teste de Shapiro-Wilk, mas os resultados revelaram que não apresentam distribuição normal ( $p$-value de 0,000 para ambas as variáveis). De acordo com Hair Jr. et al. (2011), quando os dados são medidos por escalas ordinais ou nominais, a suposição de que os dados são normais nem sempre é válida. Nestes casos, sugere-se a utilização de testes não paramétricos.

Assim, foi utilizado o teste não paramétrico de Wilcoxon-Mann-Whitney para duas amostras independentes. O teste de Wilcoxon-Mann-Whitney foi usado para determinar se as 
diferenças nos valores médios atribuídos às práticas de gestão de pessoas e a cultura de segurança são estatisticamente significativos. No teste de Wilcoxon-Mann-Whitney (U), valores de $p$-value menores que 0,05 demonstram que os grupos são significativamente diferentes.

Constata-se na Tabela 6, por intermédio da análise realizada aos pares, que as práticas de gestão de pessoas e a cultura de segurança, demonstram semelhança e ou igualdade entre os valores médios apresentados, constando assim uma uniformidade dos conceitos estabelecidos entre ambas as práticas em todas as áreas de atuação pesquisadas. O processo de modificação, adaptação e consolidação das pessoas em relação aos conceitos estabelecidos de cultura de segurança, segundo Bley (2011) é constituída essencialmente através do comportamento e atitudes das pessoas atrelados as diretrizes organizacionais reciprocamente. Evidencia-se que foram raros os componentes que apresentaram valores médios significativamente diferentes. 


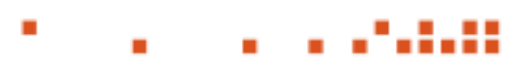 \\ Tabela 6}

Wilcoxon-Mann-Whitney Teste: Área de Trabalho Por Variável

\begin{tabular}{|c|c|c|c|c|c|c|c|c|c|c|c|c|c|c|}
\hline \multirow{4}{*}{\multicolumn{2}{|c|}{ Wilcoxon-Mann-Whitney (U teste) }} & \multicolumn{13}{|c|}{ Variáveis } \\
\hline & & \multicolumn{5}{|c|}{ Práticas de Gestão de Pessoas } & \multicolumn{8}{|c|}{ Cultura de Segurança } \\
\hline & & \multicolumn{5}{|c|}{ Componentes } & \multicolumn{8}{|c|}{ Componentes } \\
\hline & & 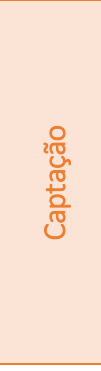 & 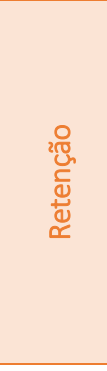 & 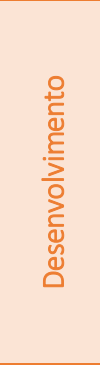 & 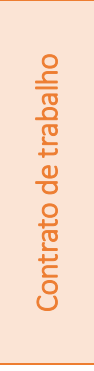 & 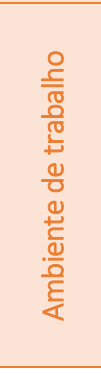 & 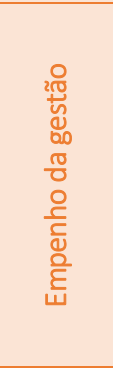 & 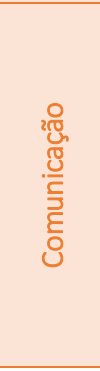 & 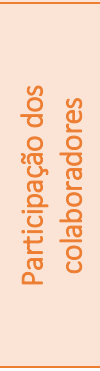 & 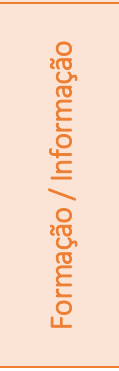 & 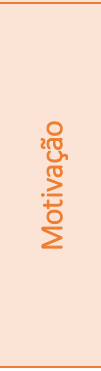 & 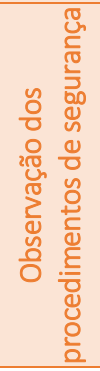 & 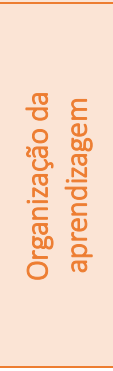 & 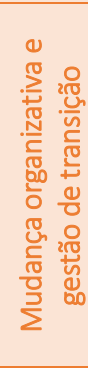 \\
\hline \multirow{3}{*}{ Administrativa e Logística } & Resultado & $\neq \mathrm{VM}$ & $\cong \mathrm{VM}$ & $\cong \mathrm{VM}$ & $\neq \mathrm{VM}$ & $\neq \mathrm{VM}$ & $\cong \mathrm{VM}$ & $\cong \mathrm{VM}$ & $\cong \mathrm{VM}$ & $\cong \mathrm{VM}$ & $\cong \mathrm{VM}$ & $\cong \mathrm{VM}$ & $\cong \mathrm{VM}$ & $\cong \mathrm{VM}$ \\
\hline & $p$-value & 0,009 & 0,055 & 0,115 & 0,019 & 0,016 & 0,335 & 0,578 & 0,771 & 0,563 & 0,926 & 0,745 & 0,565 & 0,933 \\
\hline & U teste & 225,0 & 263,0 & 284,0 & 238,5 & 234,5 & 320,0 & 344,5 & 360,5 & 343,5 & 372,5 & 358,5 & 345,5 & 373,0 \\
\hline \multirow{3}{*}{ Administrativa e Manutenção } & Resultado & $\neq \mathrm{VM}$ & $\cong \mathrm{VM}$ & $\cong \mathrm{VM}$ & $\neq \mathrm{VM}$ & $\neq \mathrm{VM}$ & $\cong \mathrm{VM}$ & $\cong \mathrm{VM}$ & $\cong \mathrm{VM}$ & $\cong \mathrm{VM}$ & $\cong \mathrm{VM}$ & $\cong \mathrm{VM}$ & $\cong \mathrm{VM}$ & $\cong V M$ \\
\hline & $p$-value & 0,001 & 0,089 & 0,137 & 0,010 & 0,017 & 0,177 & 0,809 & 0,260 & 0,183 & 0,779 & 0,235 & 0,502 & 0,187 \\
\hline & U teste & 216,0 & 325,0 & 340,0 & 269,0 & 279,5 & 349,0 & 424,5 & 364,0 & 350,5 & 422,0 & 360,0 & 397,5 & 351,5 \\
\hline \multirow{3}{*}{ Administrativa e Operacional } & Resultado & $\neq \mathrm{VM}$ & $\cong \mathrm{VM}$ & $\cong \mathrm{VM}$ & $\neq \mathrm{VM}$ & $\neq \mathrm{VM}$ & $\cong \mathrm{VM}$ & $\cong \mathrm{VM}$ & $\cong \mathrm{VM}$ & $\cong \mathrm{VM}$ & $\cong \mathrm{VM}$ & $\cong \mathrm{VM}$ & $\cong \mathrm{VM}$ & $\cong V M$ \\
\hline & $p$-value & 0,037 & 0,417 & 0,403 & 0,016 & 0,017 & 0,868 & 0,797 & 0,318 & 0,835 & 0,273 & 0,619 & 0,629 & 0,451 \\
\hline & U teste & 770,5 & 951,0 & 948,5 & 719,0 & 720,0 & 1046,5 & 1033,0 & 923,5 & 1040,5 & 911,5 & 997,5 & 1006,0 & 961,0 \\
\hline \multirow{3}{*}{ Administrativa e Florestal } & Resultado & $\cong \mathrm{VM}$ & $\cong \mathrm{VM}$ & $\cong \mathrm{VM}$ & $\cong \mathrm{VM}$ & $\cong \mathrm{VM}$ & $\cong \mathrm{VM}$ & $\cong \mathrm{VM}$ & $\cong \mathrm{VM}$ & $\cong \mathrm{VM}$ & $\cong \mathrm{VM}$ & $\cong \mathrm{VM}$ & $\cong \mathrm{VM}$ & $\cong V M$ \\
\hline & $p$-value & 0,928 & 0,333 & 0,153 & 0,243 & 0,202 & 0,403 & 0,454 & 0,334 & 0,309 & 0,377 & 0,253 & 0,149 & 0,376 \\
\hline & U teste & 30,5 & 20,5 & 15,5 & 18,5 & 17,0 & 22,0 & 23,0 & 20,5 & 20,0 & 21,5 & 18,5 & 16,0 & 21,5 \\
\hline \multirow{3}{*}{ Logística e Manutenção } & Resultado & $\cong \mathrm{VM}$ & $\cong \mathrm{VM}$ & $\cong \mathrm{VM}$ & $\cong \mathrm{VM}$ & $\cong \mathrm{VM}$ & $\cong \mathrm{VM}$ & $\cong \mathrm{VM}$ & $\cong \mathrm{VM}$ & $\cong \mathrm{VM}$ & $\cong \mathrm{VM}$ & $\cong \mathrm{VM}$ & $\cong \mathrm{VM}$ & $\cong V M$ \\
\hline & $p$-value & 0,366 & 0,888 & 0,964 & 0,906 & 0,964 & 0,680 & 0,817 & 0,317 & 0,575 & 0,796 & 0,423 & 0,953 & 0,211 \\
\hline & U teste & 668,5 & 742,0 & 751,5 & 744,5 & 751,5 & 715,0 & 733,0 & 656,5 & 700,5 & 730,5 & 676,5 & 750,5 & 633,0 \\
\hline
\end{tabular}


$. \quad . . " .+$.HE

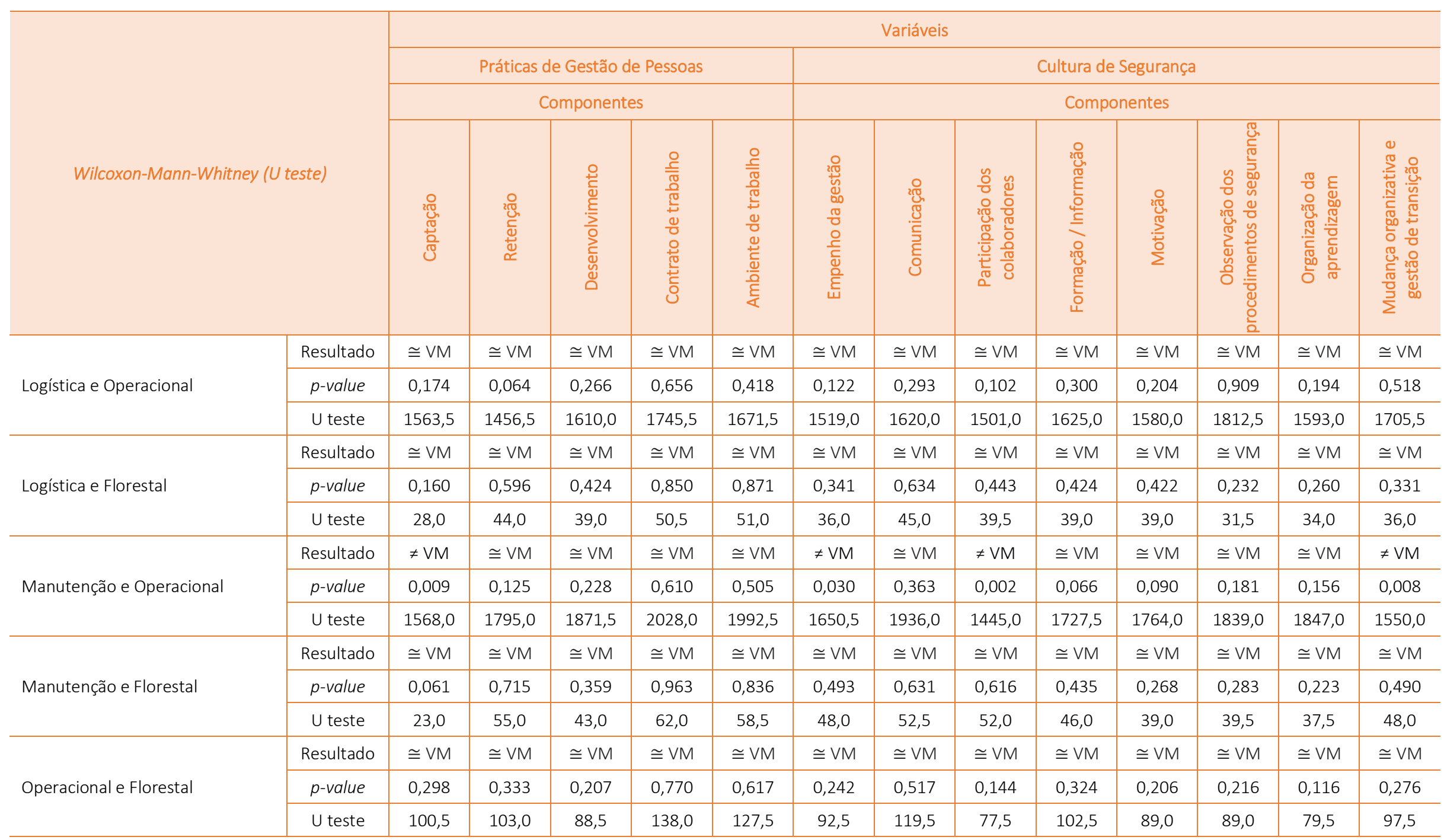

Legenda: $\neq$ (Diferença); $\cong$ (Similaridade e ou igualdade); VM (Valor médio).

Fonte: Dados da pesquisa (2020). 
A confiabilidade das variáveis foi avaliada usando o teste Alfa de Cronbach (Tabela 7). O Alfa Cronbach é uma estatística útil para a investigação da consistência interna da escala do questionário. Segundo Hair Jr. et al. (2009) para que um fator apresente consistência interna aceitável, este deve possuir Alfa de Cronbach superior a 0,600. As duas variáveis desta pesquisa apresentaram valores de boa confiabilidade dos dados com coeficientes $\alpha \geq 0,700$.

\section{Tabela 7}

Análise de Confiabilidade

\begin{tabular}{l|c|c}
\hline Variáveis & Alfa de Cronbach & № Itens \\
\hline Práticas de Gestão de Pessoas & 0,822 & 25 \\
\hline Cultura de Segurança & 0,963 & 40 \\
\hline
\end{tabular}

Fonte: Dados da pesquisa (2020).

Foram realizados os testes de Kaiser-Meyer-Olkin - Measure of Sampling Adequacy [KMO] e esfericidade Bartlett's para verificar o ajuste dos dados à Análise Fatorial [AF]. Os valores de KMO demonstram a proporção da variância que os fatores apresentam em comum, considerado o KMO mínimo de 0,6 para correta utilização da AF (Fávero et al., 2009; Hair Jr. et al., 2009). Classificado entre 0,8 e 0,9, o KMO desta pesquisa $(0,865)$ foi considerado com bom poder de explicação (Tabela 8). Baseado na distribuição estatísticas de quiquadrado para adequação do método da $A F$, o teste de esfericidade Bartlett's deve rejeitar a hipótese básica de que a matriz de correlações é identidade, apresentando valor de significância menor que 0,05 (Fávero et al., 2009; Hair Jr. et al., 2009). O teste de esfericidade Bartlett's apresentou p-value de 0,000, indicando que há correlação entre as dimensões (Tabela 8).

Tabela 8

Testes de KMO e Bartlett dos Componentes 1 a 65

\begin{tabular}{c|l|r}
\hline \multicolumn{2}{c}{ Teste de KMO e Bartlett } \\
\hline \multicolumn{2}{c}{ Medida Kaiser-Meyer-Olkin de adequação de amostragem. } &, 865 \\
\hline \multirow{2}{*}{ Teste de esfericidade de } & Aprox. Qui-quadrado & 2122,775 \\
\cline { 2 - 3 } Bartlett & Df. & 78 \\
\cline { 2 - 3 } & Sig. &, 000 \\
\hline
\end{tabular}

Fonte: Dados da pesquisa (2020).

Foram carregados cinco assertivas em cada um dos componentes da variável de gestão de pessoas. Quanto a variável cultura de segurança, foram carregados seis assertivas em Empenho da Gestão, cinco assertivas em Comunicação, seis assertivas em Participação dos Colaboradores, cinco 
assertivas em Formação e Informação, quatro assertivas em Motivação, sete assertivas em Observação dos Procedimento de Segurança, três assertivas em Organização da Aprendizagem e quatro assertivas em Mudança Organizativa e Gestão de Transição. A análise fatorial das declarações confirmou cinco componentes para a variável gestão de pessoas e oito componentes para variável cultura de segurança. A análise produziu dois fatores retratados na Tabela 9. O primeiro fator é composto em sua maioria por componentes da cultura de segurança, representando o comportamento e compromisso das pessoas e da organização com a cultura de segurança instituída; o segundo fator é constituído em sua maioria por componentes de gestão de pessoas, representando a contribuição das práticas de gestão de pessoas. Os dois fatores conjuntos neste estudo contribuíram para 68,3\% da variância total.

\section{Tabela 9}

Matriz de Componente Rotativa

\begin{tabular}{|c|c|c|c|c|}
\hline & \multicolumn{2}{|c|}{ Fatores } & \multicolumn{2}{|c|}{ Variância } \\
\hline & 1 & 2 & Variância (\%) & Acumulado (\%) \\
\hline Observação dos procedimentos de segurança & 891 & & \multirow{9}{*}{52,493} & \multirow{9}{*}{52,493} \\
\hline Motivação & ,866 & & & \\
\hline Formação / Informação & 843 & & & \\
\hline Participação dos colaboradores & 834 & & & \\
\hline Mudança organizativa e gestão de transição & 833 & & & \\
\hline Comunicação & ,799 & & & \\
\hline Empenho da gestão & ,791 & & & \\
\hline Organização da aprendizagem & ,765 & & & \\
\hline Retenção & ,656 & & & \\
\hline Ambiente de trabalho & & ,859 & \multirow{4}{*}{15,831} & \multirow{4}{*}{68,324} \\
\hline Contrato de trabalho & & ,834 & & \\
\hline Desenvolvimento & & ,751 & & \\
\hline Captação & &, 560 & & \\
\hline
\end{tabular}

Método de Extração: Análise de Componente Principal.

Método de Rotação: Varimax com Normalização de Kaiser.

a. Rotação convergida em 3 iterações.

Fonte: Dados da pesquisa (2020).

A matriz dos resíduos padronizados do instrumento de pesquisa apresentado, demonstrou que a matriz de correlações prevista não se distância da matriz de correlação observada, apresentando menos de $5 \%$ de valores fora do intervalo $[-2,58 ; 2,58]$ recomendado por Hair Jr. et al. (2009). Segundo Hair Jr. et al. (2009) a violação dessa recomendação indica deficiências no ajuste do instrumento de pesquisa estimado. A matriz dos resíduos padronizados desta pesquisa apresentou os valores do intervalo $[-2,93 ; 2,05]$, indicando ausência de problemas de ajuste no instrumento de pesquisa estimado.

Para identificar a existência, ou não, de relação entre as variáveis pesquisadas, fez-se uma análise de regressão linear múltipla para cada variável, considerando cultura de segurança como 
variável dependente e os componentes de gestão de pessoas como variáveis independentes. A regressão linear múltipla descreve a relação entre uma variável dependente (Y) e duas ou mais variáveis independentes $\left(X_{1}, X_{2}, \ldots, X_{3}\right)$, possibilitando a análise da importância concernente de cada variável do modelo, além de descrever as associações entre variáveis para melhor compreensão das correlações entre as determinantes preditoras (Hair Jr. et al., 2009).

A Tabela 10 evidencia as hipóteses de relação entre as variáveis e seus respectivos resultados.

\section{Tabela 10}

Análise de Regressão Linear Múltipla

\begin{tabular}{l|c|c|c}
\hline Hipóteses & $\beta$ & $p$-valor & Situação \\
\hline$H_{1}$ : A prática de captação influencia a cultura de segurança; & 0,002 & 0,969 & $\begin{array}{c}\text { Hipótese } \\
\text { rejeitada }\end{array}$ \\
\hline$H_{2}$ : A prática de retenção influencia a cultura de segurança; & 0,474 & 0,000 & $\begin{array}{c}\text { Hipótese não } \\
\text { rejeitada }\end{array}$ \\
\hline$H_{3}$ : A prática de desenvolvimento influencia a cultura de segurança; & 0,621 & 0,000 & $\begin{array}{c}\text { Hipótese não } \\
\text { rejeitada }\end{array}$ \\
\hline$H_{4}$ : A prática de contrato de trabalho influencia a cultura de segurança; & $-0,313$ & 0,000 & $\begin{array}{c}\text { Hipótese não } \\
\text { rejeitada }\end{array}$ \\
\hline$H_{5}:$ A prática de ambiente de trabalho influencia a cultura de segurança. & $-0,173$ & 0,009 & $\begin{array}{c}\text { Hipótese não } \\
\text { rejeitada }\end{array}$ \\
\hline
\end{tabular}

$R^{2}$ ajustado $=0,626 ; p<0,05$.

Fonte: Dados da pesquisa (2020).

Observa-se que as dimensões apresentaram relação positiva (valores próximos de $+1,0$ ) e negativa (valores próximos de -1,0) em relação aos valores de $\beta$. Segundo Hair Jr. et al. (2016), os valores de $\beta$ entre duas variáveis variam de $-1,0$ (relação negativa muito forte) a $+1,0$ (relação positiva muito forte) e, valores de $\beta$ próximos de zero demonstram relações fracas. A relação entre as dimensões Retenção $(\beta=0,474)$ e Desenvolvimento $(\beta=0,320)$ e a cultura de segurança apresentam um caráter positivo. Tais achados parecem indicar que a cultura de segurança é favoravelmente influenciada pela gestão estruturada das práticas de retenção e desenvolvimento de competências das pessoas. Desenvolvimento, das cinco práticas, é a que apresenta maior capacidade de influência. As dimensões Contrato de trabalho $(\beta=-0,313)$ e Ambiente de trabalho $(\beta=-0,173)$ apresentam um valor de beta negativo, revelando que ambas as práticas influenciam negativamente a implementação da cultura de segurança, demonstrando que organizações que não dispõem de formalização adequada das relações laborais e um ambiente favorável de trabalho, pode inviabilizar o processo de adaptação à cultura de segurança. A Tabela 10 mostra que todos os valores que testaram a relação de causa entre as dimensões apresentam-se dentro dos parâmetros propostos por Hair Jr. et al. (2016), o que confirma as hipóteses propostas nesta pesquisa, com exceção da H1. 
Vieira (2010) evidência em sua pesquisa que as práticas de gestão de pessoas contribuem de forma positiva para que as organizações desenvolvam uma cultura de segurança. Dentre as práticas de gestão de pessoas, o autor salienta que o processo de formação influencia diretamente a cultura de segurança, entretanto a prática de retenção, diferentemente dos resultados obtidos nesta pesquisa, ainda necessita de estruturação para melhor contribuir com uma cultura preventiva.

Integrada as práticas de gestão de pessoas, a cultura de segurança deve ser tratada como um modelo coerente para conceber ambientes laborais positivos e favoráveis aos colaboradores e a organização (West et al., 2011). Neste mesmo sentido, Kokkaew e Koompai (2012) declaram que as práticas provenientes da gestão de pessoas, como o desenvolvimento de competências, o desenvolvimento e a retenção dos colaboradores, está associado a ambientes de trabalho mais seguros e uma maior orientação das pessoas quanto a cultura de segurança.

Os resultados deste estudo corroboram com os de Vieira, Silva e Silva (2014), os quais concluíram que as práticas de gestão de pessoas influenciam a instituição da cultura de segurança nas organizações. Os autores destacam que a capacitação das pessoas, ressalta a importância da aprendizagem contínua, contribuindo consideravelmente para evolução da organização. Bley (2011) menciona que as práticas de gestão de pessoas devem constituídas e direcionadas para no desenvolvimento das pessoas, pois estas possuem um papel fundamental no processo evolutivo da cultura de segurança prevencionista.

De acordo com Camuffo, De Stefano e Paolino (2017) as práticas de gestão de pessoas devem ser direcionadas para ampliar o compromisso, motivação e envolvimento das pessoas, estabelecendo assim um amplo conjunto de práticas apropriadas para o desenvolvimento de cultura de segurança nas organizações, constituindo assim um ambiente de trabalho mais justo.

Munu et al. (2017) manifestam que as práticas de gestão de pessoas podem ser usadas para mitigar os riscos existentes nas organizações, concebendo uma cultura de segurança mais longeva. Os pressupostos básicos de segurança devem compor as práticas de captação, retenção e desenvolvimento de pessoas.

\section{Considerações finais}

O presente estudo teve como objetivo analisar a influência das práticas de gestão de pessoas na cultura de segurança. Mediante os resultados, constatou-se que as práticas de retenção, desenvolvimento, contrato de trabalho e ambiente de trabalho influenciam a cultura de segurança, exceto a dimensão captação.

$\mathrm{O}$ resultado do teste da hipótese $\mathrm{H} 1$ no que diz respeito a gestão de pessoas, apresentou a prática de Captação no teste confirmatório como influência moderadora inexistente para a variável 
cultura de segurança. A conclusão do teste das hipóteses $\mathrm{H} 2, \mathrm{H} 3, \mathrm{H} 4$ e H5 no que corresponde a gestão de pessoas, evidenciou as práticas de Retenção, Desenvolvimento, Contrato e Ambiente de Trabalho no teste confirmatório como influência moderadora para a variável cultura de segurança (Tabela 10).

As práticas de gestão de pessoas são imprescindíveis para uma cultura de segurança bemsucedida e duradoura. Dentre as principais atividades evidenciadas nas práticas de gestão de pessoas, pode-se destacar: capacitação, aperfeiçoamento de competências, ambiente de trabalho adequado, programas de recompensa, transparência e a legalidade dos processos legais e administrativos. Independentemente da prática de gestão de pessoas captação não ter evidenciado influência na cultura de segurança, compreendemos a importância da atividade integrativa, para melhor adaptação do recém admitido à cultura, políticas e normativas da organização.

A partir desta análise infere-se que as organizações, por intermédio da área de gestão de pessoas, devem adotar formas de abordar as questões relacionadas a cultura de segurança, utilizandose adequadamente das práticas de gestão de pessoas instituídas, com a intenção de criar abordagens direcionadas, respeitando a diversidade de perfis pessoais e profissionais, para manter e ampliar o nível de satisfação das pessoas.

Como recomendação de novos trabalhos, sugere-se a replicação deste estudo em outros segmentos mercadológicos. Afinal, os resultados encontrados nesta pesquisa não podem ser generalizados, e a sua aplicação e ampliação em outras áreas, com diferentes características contribuiria para o entendimento e amadurecimento teórico e empírico sobre o tema, posto que as práxis de gestão de pessoas encontram-se em constante evolução.

Os resultados desta pesquisa compõem contribuições para pesquisadores, profissionais, organizações, universidades e instituições de pesquisa. Este estudo fornece um entendimento para os estudiosos que desejam compreender a importância das práticas de gestão de pessoas e sua relação com a cultura de segurança.

\section{Referências}

AIEA - Agência Internacional de Energia Atômica. (1991). Safety séries nº 75 - INSAG - 4 - Safety Culture, Vienna: International Atomic Energy Agency.

Alves, J., Chagas, P. C. Z., \& Lemos, R. (2010). Processo de demissão e suas implicações: perspectivas dos gestores e dos colaboradores remanescentes em empresas de médio e grande porte de Getúlio Vargas, RS. Publicado nos Anais do $10^{\circ}$ Congresso de Stress da ISMA-BR, $12^{\circ}$ Fórum Internacional de QVT, $2^{\circ}$ Encontro Nacional de Qualidade de Vida na Segurança Pública e no Serviço Público. Vol.5 - n.11-jul./dez.

Alves, J. L. L. (2014). Comportamento de risco. Revista Proteção, n. 1. Disponível em: http://www.protecao.com.br/edicoes/1/2014/AAjj. Acesso em: novembro de 2020. 
Araújo, L. D., \& Pessoas, A. G. (2006). Estratégias e integração organizacional. São Paulo: Atlas.

Araújo, R. P., Santos, N., \& Mafra, W. J. (2006). Gestão da Segurança e Saúde do trabalho. III SEGeTSimpósio de Excelência em Gestão e Tecnologia. Rio de Janeiro/RJ.

Araújo, G. M. (2005). Avaliação da maturidade de um sistema de gestão: Proposta de um instrumento. Dissertação de Mestrado em Sistema de Gestão, Universidade Federal Fluminense - UFF, Niterói/RJ.

Azevedo, A. B. A., \& Souza, S. (2014). Os dilemas da saúde e segurança do trabalho nas Cooperativas. Revista da ABET (Online), v. 12, p. 79. Disponível em:

https://periodicos.ufpb.br/ojs/index.php/abet/article/view/18516/10419

Baylão, A. L. S., \& Rocha, A. P. S. (2014). A importância do processo de recrutamento e seleção de pessoal na organização empresarial. In: XI simpósio de excelência em gestão e tecnologia Seget 2014. Resende. XI SEGET 2014.

Batiz, E. C., dos Santos, A. F., \& Licea, O. E. A. (2009). A postura no trabalho dos operadores de checkout de supermercados: uma necessidade constante de análises. Production, 19(1), 190 201. https://doi.org/10.1590/\$0103-65132009000100012

Blair, E., \& O'Toole, M. (2010). Leading measures: Enhancing safety climate and driving safety performance. Professional Safety, 55(08), 29-34. Disponível em: https://onepetro.org/PS/article-abstract/55/08/29/33263/Leading-Measures-EnhancingSafety-Climate-and

Bley, J. (2011). Comportamento seguro: a psicologia da segurança no trabalho e a educação para a prevenção de doenças e acidentes. Versão e-book. Disponível em: www.comportamentoseguronotrabalho.blogspot.com. Acesso em: novembro de 2020.

Bohlander, G. W., \& Snell, S. A. (2009). Managing Human Resources. South-Western Cengage Learning.

Boog, G. G., \& Boog, M. T. (2008) (Coord.) Manual de Treinamento e Desenvolvimento: processo e operações. São Paulo: Pearson Prentice Hall.

Branco, R. (2008). O Movimento da Qualidade em Portugal. Grupo Editorial Vida Económica.

Brunes, L. F., \& Dias, S. B. A. (2013). O desenvolvimento profissional e a progressão de carreira estudo de caso no IPOG. Revista On-Line IPGO / Especialize - Instituto de Pós-graduação e Graduação-IPOG.

Câmara, P. B., Guerra, P. P., \& Rodrigues, J. V. (2003). Humanator: recursos humanos e sucesso empresarial. Lisboa: Edições Dom Quixote.

Campbell, G., \& Skillings, J. H. (1985). Nonparametric stepwise multiple comparison procedures. Journal of the American Statistical Association, 80(392), 998-1003. https://doi.org/10.1080/01621459.1985.10478216

Camuffo, A., De Stefano, F., \& Paolino, C. (2017). Safety reloaded: lean operations and high involvement work practices for sustainable workplaces. Journal of Business Ethics, 143(2), 245-259. https://doi.org/10.1007/s10551-015-2590-8 
Carder, B., \& Ragan, P. W. (2003). A survey-based system for safety measurement and improvement. Journal of safety research, 34(2), 157-165. https://doi.org/10.1016/S0022-4375(03)00007-0

Castellani, M. (2017). Metacoaching e Gestão Integral. Metacoaching. Disponível em: <http:// http://www.metacoaching.com.br/artigo/gestao-integral. Acesso em: novembro de 2020.

Collins, C. J., \& Smith, K. G. (2006). Knowledge exchange and combination: the role of human resource practices in the performance of hightechnology firms. Academy of Management Journal 49(3): 544-560. https://doi.org/10.5465/amj.2006.21794671

Cooper, D. (2000). Towards a model of safety culture. Safety Science, 36(2), p.111-136. https://doi.org/10.1016/S0925-7535(00)00035-7

Corrigan, S., Kay, A., Ryan, M., \& Ward, M. E. (2018). Brazil, B. Human factors and safety culture: Challenges and opportunities for the port environment. Safety Science, 119, 252-265. https://doi.org/10.1016/j.ssci.2018.03.008

Coutinho, M. L. G., Maximiano, A. C. A., \& Limongi-França, A. C. (2010). Implementation of Work Life Quality Programs with The Project Management Model. Revista de Gestão e Projetos, 1(1), 172. http://dx.doi.org/10.5585/GeP.201011

Choudhry, R. M., Fang, D., \& Mohamed, S. (2007). The nature of safety culture: A survey of the stateof-the-art. Safety science, 45(10), 993-1012. https://doi.org/10.1016/j.ssci.2006.09.003

Christopher, N. (2019). The effectiveness of HRM policies and practices. International Journal of Social Sciences, 2(1), 24-32. https://doi.org/10.31295/ijss.v2n1.51

Dejours, C. (2012). Psicodinâmica do trabalho: contribuições da escola Dejouriana à análise da relação prazer, sofrimento e trabalho. São Paulo: Atlas.

Delery, J. E., \& Roumpi, D. (2017). Strategic human resource management, human capital and competitive advantage: is the field going in circles?. Human Resource Management Journal, 27(1), 1-21. https://doi.org/10.1111/1748-8583.12137

Demo, G., Fogaça, N., \& Costa, A. C. (2018). Políticas e práticas de gestão de pessoas nas organizações: cenário da produção nacional de primeira linha e agenda de pesquisa. Cadernos EBAPE. BR, 16(2), 250-263. https://doi.org/10.1590/1679-395159073

Demo, G. (2011). Desenvolvimento e validação da Escala de Percepção de Políticas de Gestão de Pessoas (EPPGP). Revista de Administração Mackenzie, São Paulo, v.9, n.6, p.77-101. https://doi.org/10.1590/S1678-69712008000600005

Dessler, G. (2003). Administração de recursos humanos. Tradução: Cecília Leão Oderich. São Paulo: Pearson Prentice Hall, 2 ed.

Dewettinck, K., \& Remue, J. (2011). Contextualizing HRM in comparative research: The role of the Cranet network. Human Resource Management Review, 21(1), 37-49. https://doi.org/10.1016/j.hrmr.2010.09.010

Donate, M. J., Peña, I., \& Sanchez de Pablo, J. D. (2016). HRM practices for human and social capital development: effects on innovation capabilities. The International Journal of Human Resource Management, 27(9), 928-953. https://doi.org/10.1080/09585192.2015.1047393 
Dutra, J. S. (2008). Gestão de pessoas: modelo, processos, tendências e perspectivas. São Paulo: Atlas. Fávero, L. P., Belfiore, P., Silva, F. L., \& Chan, B. L. (2009). Análise de dados: modelagem multivariada para tomada de decisões. Rio de Janeiro: Elsevier.

Fiuza, G. D. (2008). Desenvolvimento e validação da escala de percepção de políticas de gestão de pessoas (EPPGP). RAM. Revista de Administração Mackenzie, 9(6), 77-101. https://doi.org/10.1590/S1678-69712008000600005

Foroni, P. G. (2014). Gestão estratégica de pessoas: um estudo de caso sobre o alinhamento estratégico. Dissertação de mestrado em Administração, Universidade de São Paulo - USP, São Paulo/SP.

Franco, J. D. O. (2012). Cargos, Salários e Remunerações. Curitiba: IESDE Brasil.

Freitas, E. (2014). A influência da Gestão de Recurso Humanos no desempenho ambiental no setor mecânico brasileiro. Tese de doutorado em Administração, Universidade Nove de Julho UNINOVE, São Paulo/SP).

Freitas, W. R. S., Jabbour, C. J. C., \& Santos, F. C. A. (2011). Continuing the evolution: towards sustainable HRM and sustainable organizations. Business Strategy Series, v. 12, n. 5, p. 226234. https://doi.org/10.1108/17515631111166861

Garcia, H., \& Garcia, D. S. S. (2016). A sentença trabalhista e o novo código de processo civil. In: II Congresso Catarinense de Direito Processual Civil, 2016, Itajaí. Anais do Congresso Catarinense de Direito Processual Civil. Itajaí: Univali, v. 2. p. 96-116. Disponível em: https://periodicos.univali.br/index.php/accdp/article/view/10178

Gonçalves, A. P. (2011). Cultura e gestão de segurança no trabalho em organizações industriais: uma proposta de modelo. Tese de doutorado em engenharia industrial, Universidade Federal da Bahia - UFBA, Salvador/BA.

Hair Jr., J. F., Black, W. C., Babin, B. J., Anderson, R. E., \& Tatham, R. L. (2009). Análise multivariada de dados. 6. ed. Porto Alegre: Bookman, 2009.

Hair Jr., J. F., Wolfinbarger, M., Money, A. H., Samoel, P., \& Page, M. J. (2011). Essentials of business research methods. Routledge, 2nd edition, 496p. ISBN-13: 978-0765626318

Helmer, G. W. (2002). Safety culture: sustaining the strategy. Occupational health \& safety (Waco, Tex.), 71(12), 14-6. Disponível em: https://www.elibrary.ru/item.asp?id=6282129

Hipólito, J. A. M. (2000). Sistema de recompensas: uma abordagem atual. Editora: Atlas.

INSAG - International Nuclear Safety Advisory Group. (1988). Safety Principles for Nuclear Power Plants. Safety Series No.75-INSAG-3, issued in 1988.

Jabbour, C. J. C., Freitas, W. R. D. S., Teixeira, A. A., \& Jabbour, A. B. L. D. S. (2012). Gestão de recursos humanos e desempenho operacional: evidências empíricas. Gestão \& Produção, 19(2), 347360. https://doi.org/10.1590/S0104-530X2012000200009 
Jabbour, C. J. C., Santos, F. C. A., \& Nagano, M. S. (2009). Análise do relacionamento entre estágios evolutivos da gestão ambiental e dimensões de recursos humanos: estado da arte e survey em empresas brasileiras. Revista de Administração-RAUSP, 44(4), 342-364. Disponível em: https://www.redalyc.org/pdf/2234/223417460005.pdf

Júnior, I. L., Morais, F. C. L., \& Teixeira, F. S. (2013). Conformidade das rotinas do departamento de pessoal de uma construtora de Coronel Fabriciano em relação à legislação trabalhista e previdenciária. In 10 Congresso Integrado de Contabillidade-Governador Valladares/MG-24 e. (Vol. 25). Disponível em: https://www.ufjf.br/congreconti/files/2013/11/A3.pdf

Khoo, N. K., Hussin, H., \& Abdullah, N. (2018). managing occupational safety and health (OSH) culture practices at small and medium (S\&M) Malaysia manufacturing sector. Journal of Human Capital Development (JHCD), 11(1), 79-90. Disponível em: https://journal.utem.edu.my/index.php/jhcd/article/view/4251/3123

Kianto, A., Sáenz, J., \& Aramburu, N. (2017). Knowledge-based human resource management practices, intellectual capital and innovation. Journal of Business Research, 81, 11-20. https://doi.org/10.1016/j.jbusres.2017.07.018

Kokkaew, N., \& Koompai, S. (2012). Current practices of human resource management (HRM) in Thai construction industry: A risk and opportunity perspective. Review of Integrative Business and Economics Research, 1(1), 1. Disponível em: http://sibresearch.org/uploads/3/4/0/9/34097180/riber_riber2012-037_1-14.pdf

Lacombe, F. J. M. (2005). Recursos humanos: princípios e tendências. 1aed. São Paulo: Saraiva.

Lange, A., \& Karawejczyk, T. T. (2014). Coaching no processo de desenvolvimento individual e organizacional. Diálogo, (25), 39-56. http://dx.doi.org/10.18316/1495

Lima, V. F., Souza, C. F. M, \& Lima, L. E. M (2018). Gestão da segurança e saúde no trabalho: ações aplicadas para redução do número de acidentes numa indústria de papel. In book: Tópicos em Adminstração - Volume 2 (pp.127-137), Edition: 1, Chapter: 13, Publisher: Editora Poisson.

Limongi-França, A. C. (2008). Práticas de recursos humanos: conceitos, ferramentas e procedimentos. Revista de Administração Contemporânea, 12(2), 583-583. https://doi.org/10.1590/S141565552008000200016

Limongi-França, A. C. (1996). Indicadores Empresariais de Qualidade de Vida no Trabalho. Tese de doutorado em administração, Universidade de São Paulo - USP, São Paulo/SP.

Lindfelt, T., Ip, E. J., Gomez, A., \& Barnett, M. J. (2018). The impact of work-life balance on intention to stay in academia: Results from a national survey of pharmacy faculty. Research in Social and Administrative Pharmacy, 14(4), pp.387-390. https://doi.org/10.1016/j.sapharm.2017.04.008

Macarenco, I. (2006). Gestão com pessoas-gestão, comunicação e pessoas: comunicação como competência de apoio para a gestão alcançar resultados humanos. Tese de doutorado em ciências da comunicação, Universidade de São Paulo - USP, São Paulo/SP.

Marras, J. P. (2011). Administração de recursos humanos: do operacional ao estratégico. 14. ed. São Paulo: Saraiva. 
McClean, E., \& Collins, C. J. (2011). High-commitment HR practices, employee effort, and firm performance: Investigating the effects of HR practices across employee groups within professional services firms. Human Resource Management, 50(3), 341-363. https://doi.org/10.1002/hrm.20429

Migueles, C., Lafraia, J. R., \& Costa, G. (2007). Criando o hábito da excelência. Editora: Qualitymark, $168 p$.

Milkovich, G. T. (2000). Administração de recursos humanos. Editora: Atlas.

Munu, N., Owusu, P. A., Kizza, R., Banadda, N., \& Kabenge, I. (2012). Towards accident-free construction sites: The role of human resource management-A review. African Journal of Environmental Economics and Management ISSN, 5(5), 328-340.

Neto, F. F. J., \& Cavalcante, J. Q. P. (2004). Manual do Direito do Trabalho. Tomo II, Ed. Lumen Juris, 2a edição.

Neves, A. (2012). Uso de indicadores chave de desempenho para avaliar sistemas de gestão. Dissertação de mestrado em gestão integrada da qualidade, ambiente e segurança, Instituto Superior de Educação e Ciências - ISEC, Lisboa/PT.

Oliveira, J. C. (2014). Evolução e Maturidade em Segurança e Saúde no Trabalho: a dificultosa transição dos estágios básicos de gestão de SST (primeiro e segundo) para os estágios mais elevados (terceiro e quarto). Belo Horizonte. Disponível em: https://eustaquiodiniz.files.wordpress.com/2014/02/evoluc3a7c3a3o-e-maturidade-em-sstfevereiro-2014.pdf. Acesso em: novembro de 2020.

Pavlov, A., Mura, M., Franco-Santos, M., \& Bourne, M. (2017). Modelling the impact of performance management practices on firm performance: interaction with human resource management practices. Production Planning \& Control, 28(5), 431-443. https://doi.org/10.1080/09537287.2017.1302614

Pidgeon, N. F. (1991). Safety culture and risk management in organizations. Journal of cross-cultural psychology, 22(1), 129-140. https://doi.org/10.1177/0022022191221009

Pranee, C. (2010). Quality of work life for sustainable development. International Journal of Organizational Innovation (Online), 2(3), 124. Disponível em: https://ijoionline.org/attachments/article/22/Microsoft\%20Word\%20\%20FINAL\%20ISSUE\%20VOL\%202\%20NUM\%203\%20WINTER\%202010.pdf\#page=124

Reason, J. (2006). Human error. 17.ed. Nova York: Cambridge University Press, 302 p.

Reason, J. (2016). Managing the risks of organizational accidents. Routledge.

Reiman, T., \& Rollenhagen, C. (2014). Does the concept of safety culture help or hinder systems thinking in safety? Accident Analysis \& Prevention, 68, 5-15. https://doi.org/10.1016/j.aap.2013.10.033

Ribas, A. (2010). Desenvolvimento individual e análise de perfil: as bases para uma alta performance. In: Ser + com T\&D: Estratégias e ferramentas de treinamento e desenvolvimento para o mundo corporativo. São Paulo: Ser Mais. 
Robbins, S. P. (2004). Fundamentos de Administração: conceitos essenciais e aplicações. 4 ed. Tradução Robert Brian Taylor, Revisão técnica Reinaldo O. da Silva. São Paulo: Pearson Prentice Hall.

Rodrigues, D. A. (2012). Formas de negociação coletiva. Conteúdo Jurídico, Brasília-DF: 16 jun. Disponível em: http://www.conteudojuridico.com.br/?artigos\&ver=2.37533\&seo=1. Acesso em: novembro de 2020.

Rodrigues, J. C. P. (1996). Como conquistar e manter talentos. Fundação Dom Cabral. Rio de Janeiro: Qualitymark, 54-67.

Rodrigues, R. S. F. (2011). As práticas da segurança e higiene do trabalho nas estruturas residenciais para idosos: motivações, dificuldades e tendências. Dissertação de mestrado em segurança e higiene no trabalho, Escola Superior de Ciências Empresariais, Instituto Politécnico de Setúbal - IPS, Setúbal/PT.

Sabino, L. A. M., \& Cunha, N. C. (2016). Remuneração e salário numa abordagem administrativa. Revista GeTeC, 5(10), p.1-11. Disponível em: https://revistas.fucamp.edu.br/index.php/getec/article/view/785

Severo, V. S. (2015). A Sentença Trabalhista e o novo CPC. Estado de Direito, Porto Alegre, 29 de julho. Disponível em: http://estadodedireito.com.br/a-sentenca-trabalhista-e-o-novo-cpc/. Acesso em: novembro de 2020.

Silva, F. M. (2013). As práticas de gestão de recursos humanos e o processo de identificação de competências coletivas: um estudo de caso na área de gestão de pessoas do centro administrativo do SICREDI. Tese de doutorado em Administração, Universidade Federal do Rio Grande do Sul - UFRGS, Porto Alegre/RS.

Silva, L. N., Nascimento, R. F., \& Botelho, M. A. S. (2013). A importância do programa de integração: um estudo da perspectiva dos gestores da empresa Lopes \& CIA. In: SEGeT-Simpósio de Excelência em Gestão e Tecnologia - Gestão e Tecnologia para a Competitividade.

Silveira, E. D. L. (2012). Socialização e integração de novos empregados na empresa: cultura organizacional. Embrapa Clima Temperado-Tese/dissertação (ALICE).

Schaurich, M. R. (2014). Configuração de Competências coletivas a partir das práticas de gestão de pessoas. Dissertação de mestrado em administração e negócios, Pontifícia Universidade Católica - PUC, Porto Alegre/RS.

Schuler, R. S., \& Jackson, S. E. (1987). Linking competitive strategies with human resource management practices. Academy of Management Perspectives, 1(3), 207-219. https://doi.org/10.5465/ame.1987.4275740

Sgourou, E., Katsakiori, P., Papaioannou, I., Goutsos, S., \& Adamides, E. (2014). A holistic framework for safety performance evaluation. Occupational Safety and Hygiene II, 75-79.

Spinelli, I. M. A. (2015). Formação, Desenvolvimento do Capital Humano e Vantagem Competitiva. Dissertação de mestrado em economia, Faculdade de Economia - FEP, Universidade do Porto, Porto/PT. 
Trentin, L., Duarte, M. A. T., \& dos Santos, A. J. (2018). Gestão de pessoas e manufatura enxuta: revisão da literatura. Produção em Foco, 8(2). https://doi.org/10.14521/P22375163.2018.0015.0009

Trentin, L., Santos, A. J., Batiz, E. C., \& Duarte, M. A. T. (2016). Qualidade de vida em uma empresa têxtil: levantamento do nível de satisfação dos funcionários. Revista ESPACIOS/ Vol. 37 (№ 07). Disponível em: https://www.revistaespacios.com/a16v37n07/16370703.html

Turner, B. A., Pidgeon, N., Blockley, D., \& Toft, B. (1989). Safety culture: its importance in future risk management. In Position paper for the second World Bank workshop on safety control and risk management, Karlstad, Sweden (pp. 6-9).

Vieira, M. A. (2010). A influência das práticas e políticas de gestão de pessoas no processo de institucionalização de uma cultura de segurança: um estudo de caso em uma organização industrial de grande porte. Dissertação de mestrado em administração, Fundação Instituto Capixaba de Pesquisas em Contabilidade Economia e Finanças - FUCAPE, Vitória/ES.

Vieira, M. A., Silva Junior, A., \& Silva, P. D. O. M. D. (2014). Influências das políticas e práticas de gestão de pessoas na institucionalização da cultura de segurança. Production, 24(1), 200-211. https://doi.org/10.1590/S0103-65132013005000022

Visser, M. (2010). Configurations of human resource practices and battlefield performance: A comparison of two armies. Human Resource Management Review, 20(4), 340-349. https://doi.org/10.1016/j.hrmr.2010.04.002

Xavier, O. (1986). Clima organizacional na pesquisa agropecuária: percepção e aspiração. Revista de Administração, v. 21, n. 4, p. 33-48, out./dez. Disponível em: https://www.alice.cnptia.embrapa.br/bitstream/doc/87375/1/Climaorganizacional.pdf

West, M., Dawson, J., Admasachew, L., \& Topakas, A. (2011). NHS staff management and health service quality: results from the NHS staff survey and related data. London: Department of Health.

Youndt, M. A., \& Snell, S. A. (2004). Human resource configurations, intellectual capital, and organizational performance. Journal of managerial issues, 337-360. Disponível em: https://www.jstor.org/stable/40604485 